\title{
Testing the neutrino mass generation mechanism at the future colliders
}

\section{Arindam Das $^{a, b, *}$}

anstitute for the Advancement of Higher Education, Hokkaido University, Sapporo 060-0817, Japan

${ }^{b}$ Department of Physics, Hokkaido University, Sapporo 060-0810, Japan

E-mail: adas@particles.sci.hokudai.ac.jp

The generation of the neutrino mass is an essential observation from the neutrino oscillation experiments. This indicates a major revision of the Standard Model which initiated with the massless neutrinos. A possible interesting scenario is the seesaw mechanism where SM gauge singlet Right Handed Neutrinos are introduced. Another interesting aspect is the extension of the SM with $\mathrm{SU}(2)_{\mathrm{L}}$ triplet fermions. Alternatively a general U(1) extension of the SM is also an interesting idea which involves three generations of the SM singlet RHNs to generate the tiny neutrino mass through the seesaw mechanism. Additionally such models can contain a $Z^{\prime}$ boson which could be tested at the colliders through the pair production of the RHNs.

*** The European Physical Society Conference on High Energy Physics (EPS-HEP2021), ***

*** 26-30 July $2021 * * *$

*** Online conference, jointly organized by Universität Hamburg and the research center DESY ***

${ }^{*}$ Speaker 


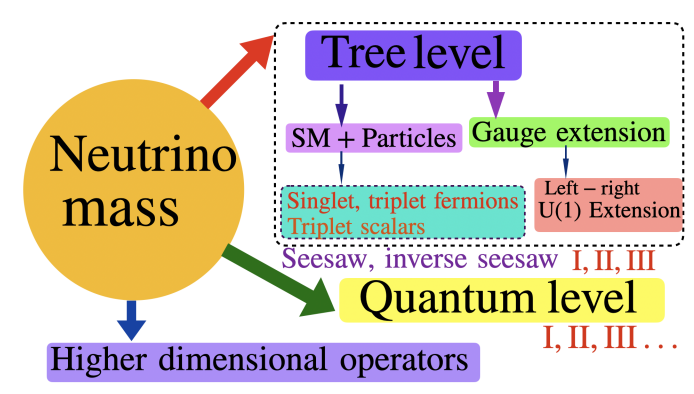

Figure 1: A graphic representation of the neutrino mass generation scenarios.

\section{Introduction}

The experimental evidence for neutrino oscillations and lepton flavor mixings, from the various experiments, motivate extensions of the SM incorporating non-zero neutrino masses and mixings. After the pioneering realization of the unique $\mathrm{d}=5$ Weinberg operator within the SM with $\Delta L=2$ lepton number violation ( $\mathrm{L}=$ Lepton number), it was realized that the very well known Seesaw mechanism could be the simplest idea to explain the smallness of the neutrino masses and flavor mixings. In many of these models, SM is extended by gauge singlet, Majorana type, heavy right handed neutrinos (RHNs). After electroweak (EW) symmetry breaking, the light Majorana neutrino masses are generated by, for instance, the so called type-I seesaw mechanism. Apart from the seesaw mechanism the SM can be extended using $\mathrm{SU}(2)_{L}$ triplet scalar, triplet fermions which are called the type-II and type-III seesaw scenarios. Apart from the tree level neutrino mass generation mechanism there is an alternative way of generating the neutrino mass at the quantum level where the neutrino masses can be explained from the one, two, three or more loop generated processes. Besides these U(1) gauge extended and Left-Right scenarios are interesting aspects where neutrino mass can be generated at the tree level and quantum level. Depending on the choice of the neutrino mass generation models LHC has started searching the heavy fermions. Firstly they considered the seesaw scenario which is extended by SM singlet heavy right handed neutrinos. A graphic representation of the possible aspects of the neutrino mass generation mechanism has been shown in Fig. 1 [1-5]. A variety of heavy neutrino and heavy triplet productions have been studied in [6-28] which investigate the limits on the heavy fermion mass and mixing plane. At the 8TeV LHC, with $20.3 \mathrm{fb}^{-1}$ luminosity and $95 \%$ confidence limit (C. L.), ATLAS [29] has probed mixings for muon flavor down to a $\left|V_{\mu N}\right|^{2}$ of $3.5 \times 10^{-3}$, for $M_{N}=100 \mathrm{GeV}$. The limits further goes down to $2.9 \times 10^{-3}$ for $M_{N}=110 \mathrm{GeV}$ and then monotonically weakens with mass, up to $M_{N}=500$ GeV. At $M_{N}=500 \mathrm{GeV}$ the limits are $\left|V_{\mu N}\right|^{2}=4 \times 10^{-1}$. The limits are nearly two orders of magnitude weaker in the case of electron flavor mixings $\left|V_{e N}\right|^{2}$ at the $95 \%$ C. L. CMS has also studied the SSDL plus di-jet signal and obtain the exclusion limits for $\left|V_{e N}\right|^{2}$ [30] and $\left|V_{\mu N}\right|^{2}$ [31]. Both studies are performed at the $8 \mathrm{TeV}$ LHC with $19.7 \mathrm{fb}^{-1}$ luminosity at $95 \% \mathrm{C}$. L. The limits for the mixed $e^{ \pm} \mu^{ \pm}+j j$ final state was also considered in (2). CMS observed upper limits for $\left|V_{e N}\right|^{2}$ at $1.2 \times 10^{-4}$ for $M_{N}=40 \mathrm{GeV}, 2 \times 10^{-2}$ for $M_{N}=85 \mathrm{GeV}, 8 \times 10^{-3}$ for $M_{N}=130 \mathrm{GeV}$ and $1.2 \times 10^{-2}$ for $M_{N}=200 \mathrm{GeV}$. Thus, the $\left|V_{e N}\right|^{2}$ limits were found to be weakened with the increase in $M_{N}$. Alternatively, RHNs may be excluded as large as $M_{N}=480 \mathrm{GeV}$, assuming the mixing 


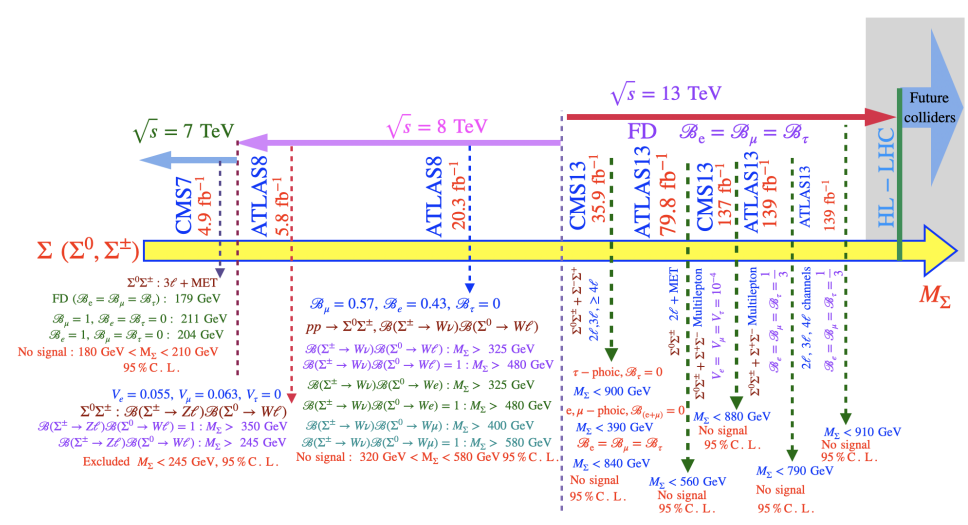

Figure 2: The mass scale of the triplet fermion and the different bounds on the triplet fermion from the LHC at $\sqrt{s}=7 \mathrm{TeV}, 8 \mathrm{TeV}$ and $13 \mathrm{TeV}$ respectively [27].

is unity. The limits on $\left|V_{\mu N}\right|^{2}$ from the SSDL + dijet final state with $\mu$ flavor is probed down to $2 \times 10^{-5}$ for $M N=40 \mathrm{GeV}, 4.5 \times 10^{-3}$ for $M_{N}=90 \mathrm{GeV}, 1.75 \times 10^{-3}$ for $M_{N}=125 \mathrm{GeV}$ and $7 \times 10^{-3}$ for $M_{N}=175 \mathrm{GeV}$ with $\left|V_{\mu N}\right|^{2}$ again weakening subsequently with the increase in $M_{N}$. For $M_{N}=500 \mathrm{GeV}$ the limit is $\left|V_{\mu N}\right|^{2}=0.6$. Apart from the seesaw scenario, $\mathrm{SU}(2)_{L}$ triplet extended fermions have been searched at the LHC and the complete roadmap of the exclusions have been shown in Fig. 2 where a recent CMS analysis rules out the possibility of $M_{\Sigma}<910 \mathrm{GeV}$ from the point of view of a flavor diagonal framework.

\section{Results}

A variety of RHN production mechanism including the photon mediated/ initiated process, we have checked that photon mediated signature is always weak $\left(M_{N}<700 \mathrm{GeV}-900 \mathrm{GeV}\right)$ and strongly dependent on the $p_{T}$ (jets/ photons) cuts $[7,8]$ using the MLM matching procedure to produce the RHNs and used the corresponding ATLAL/CMS cross sections (SSDL and/or trilepton) to constrain them in the mass mixing plane. We have also shown the component cross sections where different initial states have been considered (using the MLM matching) using the Matched cross section from the 'results.html' file of the generated events. Which shows us the effect of $p_{T}$ cuts and that is which is roughly safe between $300 \mathrm{GeV}$ to $400 \mathrm{GeV}$ of the RNN mass when $p_{T}$ (jets) $>30 \mathrm{GeV}$. Note that it has been reflected in our mass mixing plane where we could probe the RHN mass (around $300 \mathrm{GeV}$ ) with $p_{T}$ (jets) $>30 \mathrm{GeV}$ below electroweak precision bounds even at HL-LHC. We claimed that at $14 \mathrm{TeV}, p_{T}^{j}>30 \mathrm{GeV}$ will help to probe the RHNs up to $300 \mathrm{GeV}$ even if the jet initiated processes are included. We have also studied the NLO-QCD production of the RHNs at the LHC and beyond where the SSDL and $3 \ell$ final states have been explored with varied renormalization and factorization scales [9] followed by the fat jets at LHC in association with SSDL [20] and single lepton plus a fat jet at the $e^{-} e^{+}$collider[19]. The corresponding limits on the mass mixing plane are given in the upper panel of Fig. 3. We have studied the $H \rightarrow N v$ process at the LHC from the Higgs production channel and calculated the bound on the light heavy mixing from the $2 \ell 2 v$ final state $[17,18]$. We study the triplet fermion production at the $e^{-} e^{+}$ 

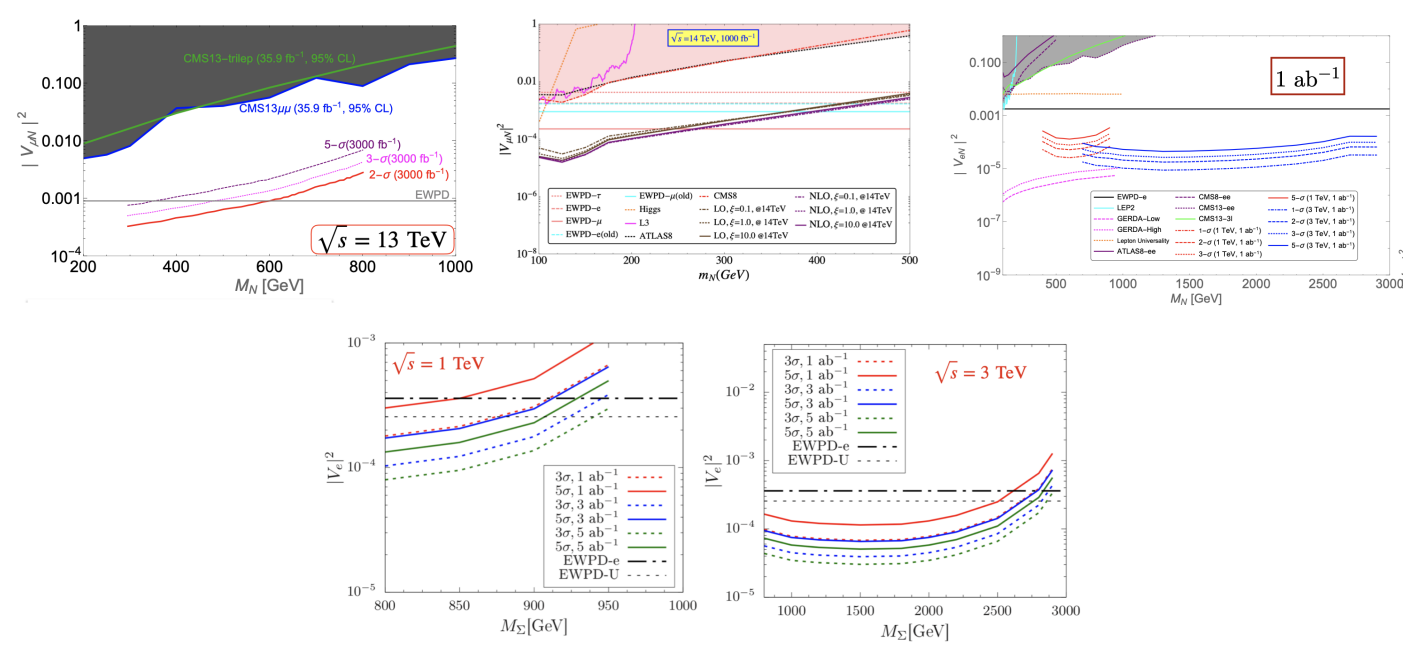

Figure 3: Upper panel: The limits on the mass mixing plane from the SSDL+ fat jet at the LHC, scale dependent NLO-QCD production of the RHNs and single lepton in association with fat jet at the $e^{-} e^{+}$collider from left to right. Lower panel: Limits on the mixing angle from the $e v+$ fat jet signal in type-III seesaw scenario.

collider where final state is $\Sigma^{+} e^{-}\left(\Sigma^{0} v\right)$ at the $s$ channel and $t$ channel respectively. We consider the triplet fermion at the $1 \mathrm{TeV}$ which will boost the decay product like SM $W$ boson into a fat jet. Hence $e v+$ fat jet signal can be probed from the triplet fermions. This process can probe the light-heavy mixing with respect to the triplet mass [26] and the corresponding limits are shown in the lower panel of the Fig. 3. The mass scale of the triplet fermion and the different bounds on the triplet fermion from the LHC at $\sqrt{s}=7 \mathrm{TeV}, 8 \mathrm{TeV}$ and $13 \mathrm{TeV}$ respectively [27] in Fig. 2.

\section{Conclusion}

We study the models with the heavy fermions under the simple extensions of the SM where the neutrino mass is generated by the seesaw mechanism at the tree level to reproduce the neutrino oscillation data. We find that such heavy fermions can be tested at the underground experimentsat the proton-proton, electron-positron and electron-proton colliders in the near future. We have calculated the bounds on the light-heavy mixings for the electron-positron collider which could be probed in the near future.

\section{References}

[1] P. Minkowski, $\mu \rightarrow$ ey at a Rate of One Out of $10^{9}$ Muon Decays?, Phys. Lett. B 67 (1977) 421-428.

[2] T. Yanagida, Horizontal gauge symmetry and masses of neutrinos, Conf. Proc. C 7902131 (1979) 95-99. 
[3] R. Foot, H. Lew, X. G. He, and G. C. Joshi, Seesaw Neutrino Masses Induced by a Triplet of Leptons, Z. Phys. C 44 (1989) 441.

[4] K. S. Babu, Model of 'Calculable' Majorana Neutrino Masses, Phys. Lett. B 203 (1988) $132-136$.

[5] R. N. Mohapatra and R. E. Marshak, Local B-L Symmetry of Electroweak Interactions, Majorana Neutrinos and Neutron Oscillations, Phys. Rev. Lett. 44 (1980) 1316-1319. [Erratum: Phys.Rev.Lett. 44, 1643 (1980)].

[6] A. Das and N. Okada, Inverse seesaw neutrino signatures at the LHC and ILC, Phys. Rev. D 88 (2013) 113001, [arXiv: 1207 . 3734].

[7] A. Das, P. S. Bhupal Dev, and N. Okada, Direct bounds on electroweak scale pseudo-Dirac neutrinos from $\sqrt{s}=8 \mathrm{TeV}$ LHC data, Phys. Lett. B 735 (2014) 364-370, [arXiv: 1405.0177].

[8] A. Das and N. Okada, Improved bounds on the heavy neutrino productions at the LHC, Phys. Rev. D 93 (2016), no. 3 033003, [arXiv: 1510.04790].

[9] A. Das, P. Konar, and S. Majhi, Production of Heavy neutrino in next-to-leading order QCD at the LHC and beyond, JHEP 06 (2016) 019, [arXiv: 1604 . 00608].

[10] A. Das and N. Okada, Bounds on heavy Majorana neutrinos in type-I seesaw and implications for collider searches, Phys. Lett. B 774 (2017) 32-40, [arXiv: 1702 . 04668].

[11] A. Das, P. S. B. Dev, and C. S. Kim, Constraining Sterile Neutrinos from Precision Higgs Data, Phys. Rev. D 95 (2017), no. 11 115013, [arXiv: 1704.00880].

[12] A. Das, P. S. B. Dev, and R. N. Mohapatra, Same Sign versus Opposite Sign Dileptons as a Probe of Low Scale Seesaw Mechanisms, Phys. Rev. D 97 (2018), no. 1015018, [arXiv: 1709.06553].

[13] A. Das, N. Okada, and D. Raut, Enhanced pair production of heavy Majorana neutrinos at the LHC, Phys. Rev. D 97 (2018), no. 11 115023, [arXiv: 1710 . 03377].

[14] A. Das, N. Okada, and D. Raut, Heavy Majorana neutrino pair productions at the LHC in minimal U(1) extended Standard Model, Eur. Phys. J. C 78 (2018), no. 9 696, [arXiv: 1711.09896].

[15] A. Das, P. S. B. Dev, and N. Okada, Long-lived TeV-scale right-handed neutrino production at the LHC in gauged U(1)X model, Phys. Lett. B 799 (2019) 135052, [arXiv: 1906. 04132].

[16] A. Das, N. Nagata, and N. Okada, Testing the 2-TeV Resonance with Trileptons, JHEP 03 (2016) 049, [arXiv: 1601.05079].

[17] A. Das, S. Jana, S. Mandal, and S. Nandi, Probing right handed neutrinos at the LHeC and lepton colliders using fat jet signatures, Phys. Rev. D 99 (2019), no. 5 055030, [arXiv: 1811.04291]. 
[18] A. Das, Searching for the minimal Seesaw models at the LHC and beyond, Adv. High Energy Phys. 2018 (2018) 9785318, [arXiv: 1803. 10940].

[19] A. Das, Pair production of heavy neutrinos in next-to-leading order QCD at the hadron colliders in the inverse seesaw framework, Int. J. Mod. Phys. A 36 (2021), no. 04 2150012, [arXiv: 1701.04946].

[20] A. Das, P. Konar, and A. Thalapillil, Jet substructure shedding light on heavy Majorana neutrinos at the LHC, JHEP 02 (2018) 083, [arXiv: 1709 . 09712].

[21] C.-W. Chiang, G. Cottin, A. Das, and S. Mandal, Displaced heavy neutrinos from Z decays at the LHC, JHEP 12 (2019) 070, [arXiv: 1908.09838].

[22] A. Bhardwaj, A. Das, P. Konar, and A. Thalapillil, Looking for Minimal Inverse Seesaw scenarios at the LHC with Jet Substructure Techniques, J. Phys. G 47 (2020), no. 7 075002, [arXiv: 1801.00797].

[23] G. Cvetič, A. Das, and J. Zamora-Saá, Probing heavy neutrino oscillations in rare $W$ boson decays, J. Phys. G 46 (2019) 075002, [arXiv: 1805 .00070].

[24] G. Cvetič, A. Das, S. Tapia, and J. Zamora-Saá, Measuring the heavy neutrino oscillations in rare W boson decays at the Large Hadron Collider, J. Phys. G 47 (2020), no. 1 015001, [arXiv: 1905.03097].

[25] A. Das, N. Okada, S. Okada, and D. Raut, Probing the seesaw mechanism at the $250 \mathrm{GeV}$ ILC, Phys. Lett. B 797 (2019) 134849, [arXiv: 1812 .11931].

[26] A. Das and S. Mandal, Bounds on the triplet fermions in type-III seesaw and implications for collider searches, Nucl. Phys. B 966 (2021) 115374, [arXiv: 2006. 04123].

[27] A. Das, S. Mandal, and T. Modak, Testing triplet fermions at the electron-positron and electron-proton colliders using fat jet signatures, Phys. Rev. D 102 (2020), no. 3 033001, [arXiv: 2005. 02267].

[28] A. Das, Y. Gao, and T. Kamon, Heavy neutrino search via semileptonic Higgs decay at the LHC, Eur. Phys. J. C 79 (2019), no. 5 424, [arXiv: 1704.00881].

[29] ATLAS Collaboration, G. Aad et al., Search for heavy Majorana neutrinos with the ATLAS detector in pp collisions at $\sqrt{s}=8 \mathrm{TeV}$, JHEP 07 (2015) 162, [arXiv: 1506.06020].

[30] CMS Collaboration, V. Khachatryan et al., Search for heavy Majorana neutrinos in ee+ jets and e $\mu+$ jets events in proton-proton collisions at $\sqrt{s}=8 \mathrm{TeV}$, JHEP 04 (2016) 169, [arXiv: 1603.02248].

[31] CMS Collaboration, V. Khachatryan et al., Search for heavy Majorana neutrinos in $\mu^{ \pm} \mu^{ \pm}+$ jets events in proton-proton collisions at $\sqrt{s}=8 \mathrm{TeV}$, Phys. Lett. B 748 (2015) 144-166, [arXiv: 1501.05566]. 